\title{
TITLE:
}

\section{Effect of Coulomb interactions on the vibronic couplings in $\left.\mathrm{C}_{260^{\wedge}-}\right\}$}

$\operatorname{AUTHOR}(S)$ :

Iwahara, Naoya; Sato, Tohru; Tanaka, Kazuyoshi

CITATION:

Iwahara, Naoya ...[et al]. Effect of Coulomb interactions on the vibronic couplings in C\{60^-\}. Journal of Chemical Physics 2012, 136(17): 174315.

ISSUE DATE:

2012-05-07

URL:

http://hdl.handle.net/2433/187991

\section{RIGHT:}

(c) 2012 American Institute of Physics. This article may be downloaded for personal use only. Any other use requires prior permission of the author and the American Institute of Physics. 


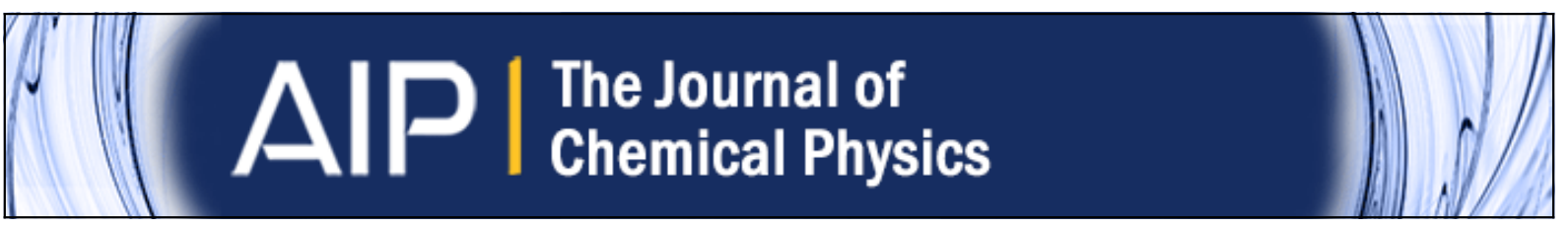

\section{Effect of Coulomb interactions on the vibronic couplings in C 60}

Naoya Iwahara, Tohru Sato, and Kazuyoshi Tanaka

Citation: The Journal of Chemical Physics 136, 174315 (2012); doi: 10.1063/1.4709611

View online: http://dx.doi.org/10.1063/1.4709611

View Table of Contents: http://scitation.aip.org/content/aip/journal/jcp/136/17?ver=pdfcov

Published by the AIP Publishing

\section{Articles you may be interested in}

The molecular structure and a Renner-Teller analysis of the ground and first excited electronic states of the jetcooled C S 2 + molecular ion

J. Chem. Phys. 124, 084312 (2006); 10.1063/1.2172612

Vibrational cooling in a cold ion trap: Vibrationally resolved photoelectron spectroscopy of cold C 60 anions J. Chem. Phys. 123, 051106 (2005); 10.1063/1.1998787

Multiphoton photodetachment of $\mathrm{C} 60$

J. Chem. Phys. 122, 064302 (2005); 10.1063/1.1844312

Lowest-energy structures of ( $\mathrm{C} 60) n X(X=\mathrm{Li}+, \mathrm{Na}+, \mathrm{K}+, \mathrm{Cl})$ and $(\mathrm{C} 60) \mathrm{n} \mathrm{Y} \mathrm{Cl}(\mathrm{Y}=\mathrm{Li}, \mathrm{Na}, \mathrm{K})$ clusters for n13

J. Chem. Phys. 121, 12315 (2004); 10.1063/1.1819894

Comment on "Group-theoretical analysis of the electronic structure data for molecular ions C $60 \mathrm{~N} \pm$ (I h ) derived from multipole expansion of the Coulomb interelectronic interactions" [J. Chem. Phys. 119, 11429 (2003)]

J. Chem. Phys. 120, 8334 (2004); 10.1063/1.1695319

\section{AlP Re-register for Table of Content Alerts}




\title{
Effect of Coulomb interactions on the vibronic couplings in $\mathrm{C}_{60}^{-}$
}

\author{
Naoya Iwahara, Tohru Sato, ${ }^{\text {a) }}$ and Kazuyoshi Tanaka \\ Department of Molecular Engineering, Graduate School of Engineering, \\ Kyoto University, Kyoto 615-8510, Japan
}

(Received 25 January 2012; accepted 17 April 2012; published online 7 May 2012)

\begin{abstract}
Vibronic couplings in $\mathrm{C}_{60}^{-}$anion are discussed on the basis of the concept of the vibronic coupling density (VCD) [T. Sato, K. Tokunaga, and K. Tanaka, J. Chem. Phys. 124, 024314 (2006); K. Tokunaga, T. Sato, and K. Tanaka, J. Chem. Phys. 124, 154303 (2006); and T. Sato, K. Tokunaga, and K. Tanaka, J. Phys. Chem. A 112, 758 (2008)]. The VCD analysis clearly reveals that the coupling to the bending $h_{g}(2)$ mode is weaker than the coupling to the stretching $h_{g}(7)$ and $h_{g}(8)$ modes. For the vibronic couplings with the stretching modes, polarizations of the electron density difference on the bonds play a crucial role in the vibronic couplings. Such a polarized electron density difference appears as a result of the Coulomb interactions between the electrons in the lowest unoccupied molecular orbital and relevant doubly-occupied orbitals. () 2012 American Institute of Physics. [http://dx.doi.org/10.1063/1.4709611]
\end{abstract}

\section{INTRODUCTION}

The vibronic coupling ${ }^{1,2}$ in the $\mathrm{C}_{60}^{-}$anion has been intensively investigated ${ }^{3-14}$ because this coupling is essential in the Jahn-Teller effect. Thus, reliable vibronic coupling constants (VCC) are required to reveal the Jahn-Teller effect of $\mathrm{C}_{60}$, and also, the role of the effect in molecular and solid state properties of fullerenes. However, vibronic couplings obtained from a photoelectron (PE) spectrum ${ }^{5}$ have been larger than the other experimental value ${ }^{9}$ and theoretical evaluations. $3,4,6,10-14$ The Jahn-Teller stabilization energy $E_{\mathrm{JT}}$ derived from the PE spectrum is $88.2 \mathrm{meV}$, while the other experimental value derived from the visible and near infrared spectrum using the $T_{1 u} \otimes h_{g}$ Jahn-Teller model is $57.9 \mathrm{meV}$ (Ref. 9) and the theoretical $E_{\mathrm{JT}}$ 's are in the range from 23.6 to $57.0 \mathrm{meV}$. Among the vibronic couplings, Gunnarsson et al. have concluded that the coupling for the bending $h_{g}(2)$ mode has the largest contribution to $E_{\mathrm{JT}}$ from the experiment. ${ }^{5} \mathrm{On}$ the other hand, many theoretical works have suggested that the stabilizations by the stretching $h_{g}(7)$ and $h_{g}(8)$ modes are the largest. ${ }^{3,10-14}$ Among the investigations on the vibronic couplings in $\mathrm{C}_{60}^{-}$, some authors have explained the order of the VCCs. ${ }^{3,8,9}$ However, the vibronic coupling whose contribution to $E_{\mathrm{JT}}$ is the largest is still controversial. Therefore, both the agreement between experimental and theoretical VCCs of $\mathrm{C}_{60}^{-}$and physical picture of the couplings are crucial to understand the vibronic couplings.

Recently, we have estimated the VCCs in $\mathrm{C}_{60}^{-}$performing the exact diagonalization of a dynamic multimode Jahn-Teller Hamiltonian with totally symmetric $a_{g}$ modes to fit the experimental PE spectrum of $\mathrm{C}_{60}^{-}$measured by Wang et al., ${ }^{15}$ and evaluated the VCCs using density-functional theory (DFT) calculations. ${ }^{16}$ The PE spectrum of Wang et al. was measured at lower temperature and with higher resolution than that of Gunnarsson et al. ${ }^{5}$ Thus, the VCCs obtained from Wang's spectrum are more reliable than Gunnarsson's constants. It

\footnotetext{
a)Electronic mail: tsato@scl.kyoto-u.ac.jp.
}

has been found that the VCCs derived from the PE spectrum of Wang et al. and the theoretical VCCs agree well with each other. Our results show that the couplings for the $h_{g}(7)$ and $h_{g}(8)$ modes are strong, while the coupling to the $h_{g}(2)$ mode is weak. The Jahn-Teller stabilizations from Wang's spectrum are from 57.7 to $65.0 \mathrm{meV}$. Theoretical $E_{\mathrm{JT}}$ 's calculated with B3LYP (Refs. 11, 13, 16, and 17) or $G W$ approximation ${ }^{14}$ are from 48.4 to $57.0 \mathrm{meV}$ and in line with the experimental ones. ${ }^{16}$ On the other hand, theoretical stabilizations calculated with local-density approximation (LDA) or generalised gradient approximation (GGA) functionals tend to be smaller than the experimental $E_{\mathrm{JT}}$ 's. ${ }^{10,12,17}$ In the previous work, we have solved the disagreement between experimental and theoretical VCCs.

The purpose of this article is to discuss the order of the VCCs of $\mathrm{C}_{60}^{-}$from another aspect, the relation between the electronic and vibrational structures. To this end, a concept of vibronic coupling density (VCD) is employed. ${ }^{18-21}$ The VCD whose integral over 3D space yields a VCC is defined from the electronic and vibrational structures. Since the VCD is a function of the position $\mathbf{r}$ in a molecule, it provides a local picture of the vibronic coupling. Employing the VCD analysis, we have succeeded in designing for novel carriertransporting materials. ${ }^{22,23}$ Furthermore, with the use of the VCD analysis, we have revealed a crucial role of the Coulomb interaction in the VCCs of hole-transporting molecules such as N,N'-diphenyl-N,N'-di(m-tolyl)benzidine (TPD) and 2,7-bis(phenyl-m-tolylamino)fluorene (TPF). ${ }^{24,25}$

\section{THEORY}

\section{A. Vibronic coupling constant}

As a reference system, the neutral $\mathrm{C}_{60}$ in its equilibrium geometry $\mathbf{R}_{0}$ with $I_{h}$ symmetry was adopted as in Ref. 16 . We use the convention of the molecular orientation by Boyle and Parker $^{26}$ (Fig. 1). The irreducible representation of the ground electronic state of $\mathrm{C}_{60}^{-}$is $T_{1 u}$. From the selection rule, 


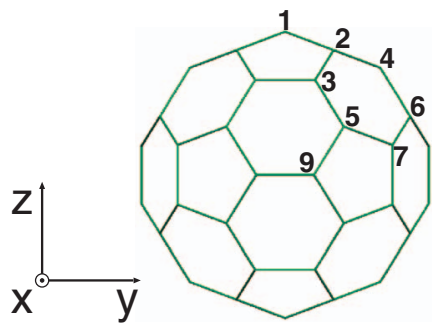

FIG. 1. The orientation and the atomic labelling of $\mathrm{C}_{60}$.

the ground electronic state couples to two $a_{g}$ and eight $h_{g}$ modes. The VCCs of $\mathrm{C}_{60}^{-}$for the $\Gamma$ modes $\left(\Gamma=a_{g}, h_{g}\right)$ are defined by ${ }^{27,28,34}$

$$
\begin{gathered}
V_{a_{g}(i)}=\left\langle\Psi_{T_{1 u} \lambda}\left|\left(\frac{\partial \hat{H}}{\partial Q_{a_{g}(i)}}\right)_{\mathbf{R}_{0}}\right| \Psi_{T_{1 u} \lambda}\right\rangle, \\
V_{h_{g}(\mu)}=\left\langle\Psi_{T_{1 u} z}\left|\left(\frac{\partial \hat{H}}{\partial Q_{h_{g}(\mu) \theta}}\right)_{\mathbf{R}_{0}}\right| \Psi_{T_{1 u} z}\right\rangle,
\end{gathered}
$$

where $\hat{H}$ is the electronic Hamiltonian which includes the Coulomb potential between nuclei. $\left|\Psi_{T_{1 u} \lambda}\right\rangle(\lambda=x, y, z)$ denotes the electronic ground state of $\mathrm{C}_{60}^{-}$at the geometry of the reference system $\mathbf{R}_{0}$, and $Q_{\Gamma(\mu) \gamma}$ is the normal coordinate of the neutral $\mathrm{C}_{60}$ for the $\Gamma(\mu) \gamma$ mode $\left(\Gamma \gamma=a_{g}, h_{g} \theta, h_{g} \epsilon, h_{g} \xi\right.$, $\left.h_{g} \eta, h_{g} \zeta\right)$. Among the three electronic states $\lambda=x, y, z$, we choose the $T_{1 u} z$ electronic state because the electronic state couples only to the $h_{g} \theta$ modes. ${ }^{29}$ Hereafter we will present results for the $T_{1 u} z$ electronic state and the $h_{g} \theta$ vibrational modes. The phase of the $a_{g}$ and $h_{g} \theta$ modes are chosen so that the VCCs are negative. Applying the Hellmann-Feynman theorem, we obtain

$$
\begin{gathered}
V_{a_{g}(i)}=\sum_{A=1}^{60}\left(\frac{\partial E(\mathbf{R})}{\partial \mathbf{R}_{A}}\right)_{\mathbf{R}_{0}} \cdot \frac{\mathbf{u}_{A}^{a_{g}(i)}}{\sqrt{M}}, \\
V_{h_{g}(\mu)}=\sum_{A=1}^{60}\left(\frac{\partial E(\mathbf{R})}{\partial \mathbf{R}_{A}}\right)_{\mathbf{R}_{0}} \cdot \frac{\mathbf{u}_{A}^{h_{g}(\mu) \theta}}{\sqrt{M}},
\end{gathered}
$$

where $E(\mathbf{R})=\left\langle\Psi_{T_{1 u} z}|\hat{H}| \Psi_{T_{1 u} z}\right\rangle, A$ denotes an atom in $\mathrm{C}_{60}, \mathbf{R}_{A}$ the Cartesian coordinates of the atom $A, \mathbf{R}$ the set of all coordinates of $\mathbf{R}_{A}, \mathbf{u}_{A}^{\Gamma(\mu) \gamma}$ the displacement of the atom $A$ of the mass-weighted $\Gamma \gamma$ vibrational vector, and $M$ the mass of carbon. For the calculation of the VCCs, we used Eqs. (3) and (4). (a)

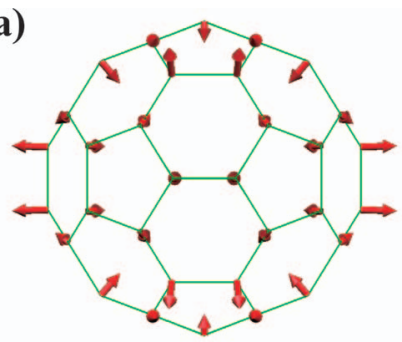

(b)

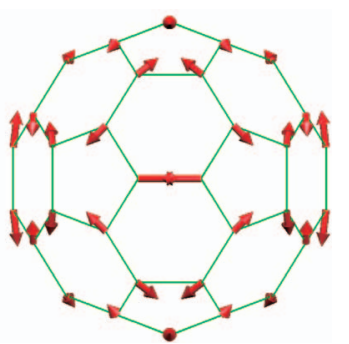

FIG. 2. The $\theta$ elements of the (a) $h_{g}(2)$ and (b) $h_{g}(8)$ modes. The orientation of $\mathrm{C}_{60}$ is the same as that of Fig. 1 .
The VCCs are decomposed into couplings for the atoms

$$
\begin{gathered}
V_{a_{g}(i), A}=\left(\frac{\partial E(\mathbf{R})}{\partial \mathbf{R}_{A}}\right)_{\mathbf{R}_{0}} \cdot \frac{\mathbf{u}_{A}^{a_{g}(i)}}{\sqrt{M}}, \\
V_{h_{g}(\mu), A}=\left(\frac{\partial E(\mathbf{R})}{\partial \mathbf{R}_{A}}\right)_{\mathbf{R}_{0}} \cdot \frac{\mathbf{u}_{A}^{h_{g}(\mu) \theta}}{\sqrt{M}} .
\end{gathered}
$$

We call $V_{a_{g}(i), A}$ and $V_{h_{g}(\mu), A}$ atomic vibronic coupling constants (AVCC).

With the use of the VCCs $V_{a_{g}(i)}$ and $V_{h_{g}(\mu)}$, the stabilization energies for the $a_{g}$ modes and the $h_{g}$ modes are written as follows:

$$
\begin{gathered}
E_{s}=\sum_{i=1}^{2} \frac{V_{a_{g}(i)}^{2}}{2 \omega_{a_{g}(i)}^{2}}, \\
E_{\mathrm{JT}}=\sum_{\mu=1}^{8} \frac{V_{h_{g}(\mu)}^{2}}{2 \omega_{h_{g}(\mu)}^{2}} .
\end{gathered}
$$

\section{B. Vibronic coupling density}

The VCCs can be rewritten as the integral form using electronic and vibrational structures. ${ }^{20,21}$ For the $h_{g}$ modes, the VCCs are written as

$$
V_{h_{g}(\mu)}=\int d^{3} \mathbf{r} \eta_{h_{g}(\mu)}(\mathbf{r}) .
$$

The vibronic coupling density $\eta_{h_{g}(\mu)}$ is defined by the product of the electron density difference $\Delta \rho$ and the potential derivative $v_{h_{g}(\mu)}$ :

$$
\eta_{h_{g}(\mu)}(\mathbf{r})=\Delta \rho(\mathbf{r}) \times v_{h_{g}(\mu)}(\mathbf{r}),
$$

where the electron density difference $\Delta \rho$ is the difference between the electron density $\rho$ of $\mathrm{C}_{60}^{-}$and the electron density $\rho_{0}$ of $\mathrm{C}_{60}$

$$
\Delta \rho(\mathbf{r})=\rho(\mathbf{r})-\rho_{0}(\mathbf{r}) .
$$

The potential derivative $v_{h_{g}(\mu)}$ is the derivative of the oneelectron potential $u(\mathbf{r} ; \mathbf{R})$ acting on a single electron from all the nuclei of $\mathrm{C}_{60}$ with respect to $Q_{h_{g}(\mu) \theta}$ :

$$
\begin{gathered}
v_{h_{g}(\mu)}(\mathbf{r})=\left(\frac{\partial u(\mathbf{r} ; \mathbf{R})}{\partial Q_{h_{g}(\mu) \theta}}\right)_{\mathbf{R}_{0}}, \\
=\sum_{A=1}^{60} \frac{-Z\left(\mathbf{r}-\mathbf{R}_{0, A}\right)}{\left|\mathbf{r}-\mathbf{R}_{0, A}\right|^{3}} \cdot \frac{\mathbf{u}_{A}^{h_{g}(\mu) \theta}}{\sqrt{M}},
\end{gathered}
$$

where $u$ is

$$
u(\mathbf{r} ; \mathbf{R})=\sum_{A=1}^{60} \frac{-Z}{\left|\mathbf{r}-\mathbf{R}_{A}\right|} .
$$

Here $Z=6$, the atomic number of carbon atom. In the present treatment, core and valence electrons are not treated separately. They are taken into account in the electron density difference $\Delta \rho$. From the VCD, we obtain a local picture of the vibronic coupling constant. 


\section{Contribution of each orbital to the electron density difference}

Within the Kohn-Sham method, $\Delta \rho$ is written as follows:

$$
\Delta \rho(\mathbf{r})=\sum_{\nu \Lambda \lambda \sigma}\left(N_{\Lambda(v) \lambda \sigma} \psi_{\Lambda(v) \lambda \sigma}^{2}(\mathbf{r})-N_{0, \Lambda(v) \lambda \sigma} \psi_{0, \Lambda(v) \lambda}^{2}(\mathbf{r})\right),
$$

where $\left\{\psi_{\Lambda(v) \lambda \sigma}\right\}$ is a set of Kohn-Sham orbitals of the $\mathrm{C}_{60}^{-}$ anion, $\left\{\psi_{0, \Lambda(v) \lambda}\right\}$ a set of the orbitals of the neutral $\mathrm{C}_{60}, \Lambda$ the irreducible representation, $\lambda$ the component of $\Lambda, \sigma$ the $z$ component of the electron spin, $v$ the quantum number except for $\Lambda, \lambda$, and $\sigma$, and $N_{\Lambda(v) \lambda \sigma}$ and $N_{0, \Lambda(v) \lambda \sigma}$ the occupation numbers of the orbitals $\psi_{\Lambda(\mu) \lambda \sigma}$ and $\psi_{0, \Lambda(\mu) \lambda}$, respectively. The Kohn-Sham orbitals are chosen to be real. In order to evaluate the contribution of each orbital to the electron density difference, we expand $\Delta \rho$ in terms of $\left\{\psi_{0, \Lambda(v) \lambda}\right\}$ :

$$
\begin{gathered}
\Delta \rho(\mathbf{r})=\sum_{\nu \Lambda \lambda \sigma} A(\Lambda(v) \lambda \sigma) \psi_{0, \Lambda(v) \lambda}^{2}(\mathbf{r}) \\
+\sum_{\nu \Lambda \lambda v^{\prime} \Lambda^{\prime} \lambda^{\prime} \sigma} B\left(\Lambda(v) \lambda \Lambda^{\prime}\left(v^{\prime}\right) \lambda^{\prime} \sigma\right) \\
\times \psi_{0, \Lambda(v) \lambda}(\mathbf{r}) \psi_{0, \Lambda^{\prime}\left(v^{\prime}\right) \lambda^{\prime}}(\mathbf{r}) \\
A(\Lambda(v) \lambda \sigma)=\sum_{\bar{v} \bar{\Lambda} \bar{\lambda}} N_{\bar{\Lambda}(\bar{v}) \bar{\lambda} \sigma}\left\langle\psi_{0, \Lambda(v) \lambda} \mid \psi_{\bar{\Lambda}(\bar{v}) \bar{\lambda} \sigma}\right\rangle^{2}-N_{0, \Lambda(v) \lambda \sigma} \\
B\left(\Lambda(v) \lambda \Lambda^{\prime}\left(v^{\prime}\right) \lambda^{\prime} \sigma\right)= \\
\sum_{\bar{\nu} \bar{\Lambda} \bar{\lambda}} N_{\bar{\Lambda}(\bar{v}) \bar{\lambda} \sigma}\left\langle\psi_{0, \Lambda(v) \lambda} \mid \psi_{\bar{\Lambda}(\bar{v}) \bar{\lambda} \sigma}\right\rangle \\
\times\left\langle\psi_{0, \Lambda^{\prime}\left(v^{\prime}\right) \lambda^{\prime}} \mid \psi_{\bar{\Lambda}(\bar{v}) \bar{\lambda} \sigma}\right\rangle
\end{gathered}
$$

\section{METHOD OF CALCULATION}

To obtain the electronic states, the equilibrium geometry $\mathbf{R}_{0}$, and the vibrational modes, DFT calculations were performed. In the DFT calculations, we employed the hybrid functional B3LYP (Ref. 30) with a triple-zeta basis set 6-311G(d). The gradients of $E(\mathbf{R})$ were calculated analytically for both a symmetrized wavefunction $\left|\Psi_{T_{1 u} z}^{\mathrm{S}}\right\rangle$ and a symmetry-broken wavefunction $\left|\Psi_{T_{1 u} z}^{\mathrm{BS}}\right\rangle$ which comes from the $z$ component of the $T_{1 u}$ state. The vibrational modes employed are symmetrized. For the DFT calculations, we used GAUSSIAN 09. ${ }^{31}$ The VCCs and VCDs were calculated using our code.

\section{RESULTS AND DISCUSSION}

\section{A. Calculation of the vibronic coupling constants}

Experimental $^{5,16,32,35}$ and present theoretical VCCs are shown in Table I. Present theoretical couplings with the symmetry-broken wavefunction $\left|\Psi_{T_{1 u z}}^{\mathrm{BS}}\right\rangle$ (Table I, BS) are in line with our couplings derived from the experimental PE spectrum of Wang et al. ${ }^{16}$ On the other hand, in comparison with the experimental VCCs, some of the VCCs calcu-

\begin{tabular}{|c|c|c|c|c|c|}
\hline & \multirow{2}{*}{$\begin{array}{l}\text { Freq. } \\
\left(\mathrm{cm}^{-1}\right)\end{array}$} & \multicolumn{2}{|c|}{ PE spectra } & \multicolumn{2}{|c|}{ B3LYP } \\
\hline & & Iwahara $^{16}$ & Gunnarsson ${ }^{5}$ & BS & S \\
\hline$a_{g}(1)$ & 496 & 0.537 & 0.15 & 0.301 & 0.308 \\
\hline$a_{g}(2)$ & 1470 & 1.644 & 2.33 & 2.352 & 2.396 \\
\hline$h_{g}(1)$ & 273 & 0.215 & 0.36 & 0.185 & 0.128 \\
\hline$h_{g}(2)$ & 437 & 0.458 & 0.84 & 0.435 & 0.448 \\
\hline$h_{g}(3)$ & 710 & 0.837 & 0.77 & 0.740 & 0.655 \\
\hline$h_{g}(4)$ & 774 & 0.628 & 0.99 & 0.554 & 1.163 \\
\hline$h_{g}(5)$ & 1099 & 0.992 & 1.15 & 0.758 & 0.211 \\
\hline$h_{g}(6)$ & 1250 & 1.010 & 0.85 & 0.544 & 0.111 \\
\hline$h_{g}(7)$ & 1428 & 2.283 & 1.78 & 2.096 & 1.783 \\
\hline$h_{g}(8)$ & 1575 & 1.581 & 2.29 & 2.031 & 2.110 \\
\hline$E_{s}$ & & 15.9 & 17.0 & 19.2 & 19.9 \\
\hline$E_{\mathrm{JT}}$ & & 57.7 & 88.2 & 49.4 & 51.0 \\
\hline$E_{s}+E_{\mathrm{JT}}$ & & 73.6 & 105.2 & 68.6 & 70.9 \\
\hline
\end{tabular}

TABLE I. Vibronic coupling constants to the $a_{g}$ and $h_{g}$ modes $\left(10^{-4}\right.$ a.u.) and the stabilization energies (meV). The VCCs obtained from photoelectron (PE) spectra are taken from Refs. 5 and 16. Experimental frequencies are taken from the data of a Raman scattering measurement of fullerite. ${ }^{36}$ The VCCs with BS and S were calculated using the symmetry-broken wavefunction $\left|\Psi_{T_{1 u} z}^{\mathrm{BS}}\right\rangle$ and symmetrized wavefunction $\left|\Psi_{T_{1 u}}^{\mathrm{S}}\right\rangle$, respectively.

lated with the symmetrized wavefunction $\left|\Psi_{T_{1 u} z}^{\mathrm{S}}\right\rangle$ (Table I, S) are qualitatively different; the VCC for the $h_{g}(4)$ mode is overestimated and the VCCs for the $h_{g}(5), h_{g}(6)$, and $h_{g}(7)$ are underestimated. As we will discuss in Sec. IV B, this discrepancy occurs because the orbital relaxation is not fully included in $\left|\Psi_{T_{1 u} z}^{\mathrm{S}}\right\rangle$. The effect of orbital relaxation is not included correctly as long as we use a symmetrized Slater determinant. Hereafter, we concentrate on the VCCs calculated with $\left|\Psi_{T_{1 u}}^{\mathrm{BS}}\right\rangle$ and analyze them.

Our theoretical VCCs for the $h_{g}(2)$ mode is almost the half of Gunnarsson's constant. Consequently the contribution of the $h_{g}(2)$ mode to the Jahn-Teller stabilization energy $E_{\mathrm{JT}}$ (8) is not the strongest, which is qualitatively consistent with many theoretical studies. ${ }^{3,10-14}$

In order to obtain the contribution to the VCCs from each atom, we calculated the AVCCs (Eqs. (5) and (6)). The AVCCs are tabulated in Table II. In general, the AVCCs for the $h_{g}(2)$ mode are smaller than those for the $h_{g}(7)$ and $h_{g}(8)$ modes. In the case of the stretching $h_{g}(7)$ and $h_{g}(8)$ modes, the AVCC is the largest at the C8 atom which is one of the $6: 6$ carbons (Fig. 1).

\section{B. Vibronic coupling density analysis}

The strengths of the vibronic couplings can be explained in terms of the VCD (9). The difference between the AVCCs for the $h_{g}(2)$ and the $h_{g}(8)$ modes (Fig. 2) is the largest at the C8 atom (Table II), thus we focus on these two modes in the vibronic coupling density analysis.

The $t_{1 u} z$ lowest unoccupied molecular orbital (LUMO) of $\mathrm{C}_{60}$ and the electron density differences of $\mathrm{C}_{60}^{-}$obtained using the symmetry-broken wavefunction $\left|\Psi_{T_{1 u} \mathrm{BS}}^{\mathrm{BS}}\right\rangle$ and the symmetrized wavefunction $\left|\Psi_{T_{1 u} z}^{\mathrm{S}}\right\rangle$ are shown in Fig. 3. The positive (gray) area of electron density difference $\Delta \rho$ originates from the LUMO density. One should note that $\Delta \rho$ (Fig. 3(b)) 
TABLE II. Atomic vibronic coupling constants of the $a_{g}$ and $h_{g}$ modes ( $10^{-4}$ a.u.). The atomic label is shown in Fig. 1 .

\begin{tabular}{|c|c|c|c|c|c|c|c|c|c|c|}
\hline Atom & $a_{g}(1)$ & $a_{g}(2)$ & $h_{g}(1)$ & $h_{g}(2)$ & $h_{g}(3)$ & $h_{g}(4)$ & $h_{g}(5)$ & $h_{g}(6)$ & $h_{g}(7)$ & $h_{g}(8)$ \\
\hline 1 & -0.004 & 0.014 & -0.010 & 0.004 & -0.003 & -0.001 & 0.004 & 0.009 & -0.028 & 0.005 \\
\hline 2 & 0.003 & -0.015 & 0.009 & -0.007 & -0.001 & 0.009 & -0.026 & 0.006 & 0.012 & -0.001 \\
\hline 3 & -0.011 & -0.090 & -0.048 & -0.044 & 0.021 & -0.063 & -0.093 & 0.065 & -0.028 & -0.098 \\
\hline 4 & -0.005 & 0.002 & -0.018 & 0.008 & -0.005 & -0.027 & 0.006 & -0.036 & -0.006 & -0.006 \\
\hline 5 & -0.027 & -0.061 & 0.042 & 0.030 & -0.051 & 0.019 & 0.069 & -0.038 & -0.039 & -0.072 \\
\hline 6 & 0.008 & -0.041 & 0.012 & -0.021 & -0.018 & 0.032 & -0.079 & -0.018 & -0.059 & 0.010 \\
\hline 7 & 0.002 & -0.031 & -0.007 & -0.003 & -0.013 & -0.014 & -0.013 & -0.103 & -0.057 & -0.004 \\
\hline 8 & -0.031 & -0.118 & 0.002 & -0.019 & -0.014 & -0.057 & 0.074 & 0.103 & -0.132 & -0.193 \\
\hline 9 & 0.019 & -0.012 & -0.017 & -0.020 & -0.035 & 0.006 & 0.003 & 0.000 & -0.010 & 0.022 \\
\hline Total & -0.301 & -2.352 & -0.185 & -0.435 & -0.740 & -0.554 & -0.758 & -0.544 & -2.096 & -2.031 \\
\hline
\end{tabular}

has not only positive but also polarized negative (blue) area. This negative area appears due to the Coulomb repulsion between the electron in the $t_{1 u}$ LUMO and the other electrons in doubly-occupied orbitals. Polarization in $\Delta \rho$ has been reported in other $\pi$ conjugated system. ${ }^{20}$

In Fig. 4, the potential derivatives and the VCDs for the $h_{g}(2)$ and $h_{g}(8)$ modes are shown. The potential derivative of the bending $h_{g}(2)$ mode $\left(v_{h_{g}(2)}\right)$ is localized on each atom (Fig. 4 (a1)). On the C1, C3, C4, C7, C8, and C9 atoms, the displacements of the $h_{g}(2)$ modes are perpendicular to the surface of the $\mathrm{C}_{60}$ cage, and the signs of $v_{h_{g}(2)}$ of the inside and outside of the cage are opposite. The VCD $\eta_{h_{g}(2)}$ is localized on each atom (Fig. 4 (a2)), and hence the integral of the VCD is canceled around the atom. In the case of the stretching $h_{g}(8)$ mode, on the other hand, $v_{h_{g}(8)}$ is delocalized on the C-C bonds of the 6:6 carbons (Fig. 4 (b1)). Since both $\Delta \rho$ and $v_{h_{g}(8)}$ are particularly delocalized on the $\mathrm{C} 8-\mathrm{C} 8^{\prime}$ bonds, $\eta_{h_{g}(8)}$ is also delocalized on the bonds (Fig. 4 (b2)). Therefore, the AVCC of the $\mathrm{C} 8$ atom is the largest. The delocalized $\eta_{h_{g}(8)}$ on the $\mathrm{C} 8-\mathrm{C}^{\prime}$ bonds appears because of the polarized electron density difference. Therefore the polarization of $\Delta \rho$ is crucial to evaluate the vibronic coupling constants. In fact, the orbital $\operatorname{VCC}\left\langle\psi_{0, \mathrm{~L} z}\left|v_{h_{g}(8) \theta}\right| \psi_{0, \mathrm{~L} z}\right\rangle$ is $-0.775 \times 10^{-4}$ a.u. where $\psi_{0, \mathrm{~L} z}$ is the LUMO of the neutral $\mathrm{C}_{60}$.

The electron density difference calculated using the symmetrized electronic state $\left|\Psi_{T_{1 u} z}^{\mathrm{S}}\right\rangle$ (Fig. 3(c)) has small negative density. Moreover, $\Delta \rho$ has negative density on the $\mathrm{C} 1, \mathrm{C} 2$, and C9 atoms despite the small LUMO coefficients on these atoms. This result suggests that the Coulomb repulsion is not fully included in the electron density difference. The distribution of $\Delta \rho$ obtained from $\left|\Psi_{T_{1 u} z}^{\mathrm{S}}\right\rangle$ is small on the C8-C8' bonds, and the VCD for the $h_{g}(8)$ is also small on the bonds. ${ }^{33}$ The discrepancy between the experimental VCCs and theoretical ones computed with $\left|\Psi_{T_{1 u} z}^{\mathrm{S}}\right\rangle$ originates from the electron density difference which does not include the orbital relaxation.

Some authors have explained the order of the VCCs of $\mathrm{C}_{60}^{-} \cdot{ }^{3,8,9}$ Varma et al. considered that the contributions of the couplings for the stretching $h_{g}(7)$ and $h_{g}(8)$ modes to $E_{\mathrm{JT}}$ are larger than that of the coupling for the bending $h_{g}(2)$ mode because for a normalized displacement the distortion along the stretching mode gives larger change in energy than that along the bending mode does. ${ }^{3}$ However, since they explained the VCC using the force constant, their discussion will not always give a correct result. Hands et al. assumed that the vibronic coupling is strong when the overlap between the $t_{1 u}$ LUMO and the displacements of the atoms is large. ${ }^{8,9}$ They concluded that the coupling for the $h_{g}(2)$ mode has larger contribution to $E_{\mathrm{JT}}$. Nonetheless, they did not take into account the derivative of the Coulomb potential between electron and nuclei $v(\mathbf{r})$, and that leads to an opposite result to ours.

\section{Electron-electron interactions in the electron density difference}

In order to evaluate the contribution from each KohnSham orbital to $\Delta \rho$, we decomposed $\Delta \rho$ into orbital densities (16). Figure 5 shows the contribution of each Kohn-Sham orbital to $\Delta \rho\left(\sum_{\lambda} \sum_{\sigma=\uparrow, \downarrow} A(\Lambda(\nu) \lambda \sigma)\right)$. Since one of the triply degenerate $t_{1 u}$ orbitals is occupied by an electron and the positive density of $\Delta \rho$ mainly originates from the $t_{1 u}(7)$ orbital, the contribution of the LUMO $\left(t_{1 u}(7)\right)$ is the largest. (a)

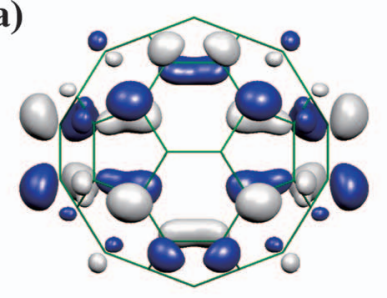

(b)

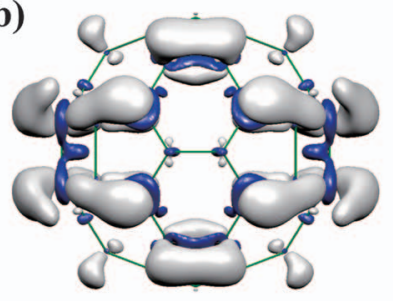

(c)

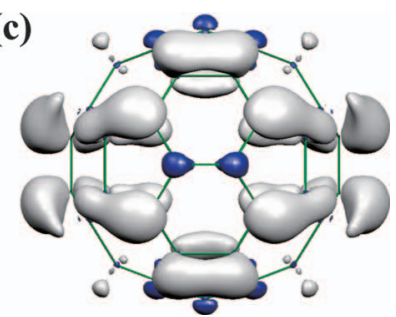

FIG. 3. (a) The LUMO, (b) the electron density difference $\Delta \rho$ calculated with the symmetry-broken wavefunction $\left|\Psi_{T_{1 u}}^{\mathrm{BS}}\right\rangle$, and (c) the electron density difference calculated with the symmetrized wavefunction $\left|\Psi_{T_{1 u} z}^{\mathrm{S}}\right\rangle$. The gray and blue indicate positive and negative, respectively. The isosurface value is 0.035 a.u. for LUMO and 0.0008 a.u for electron density difference. The orientation of $\mathrm{C}_{60}$ is the same as that of Fig. 1. 
(a1)

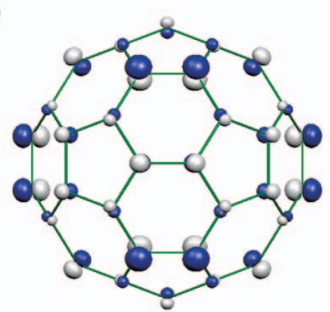

(a2)

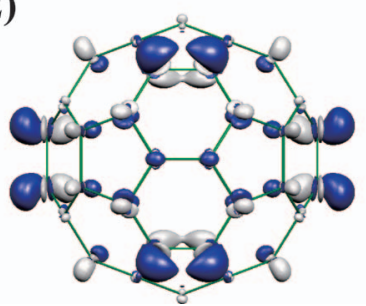

(b1)

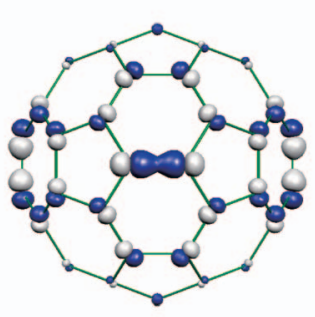

(b2)

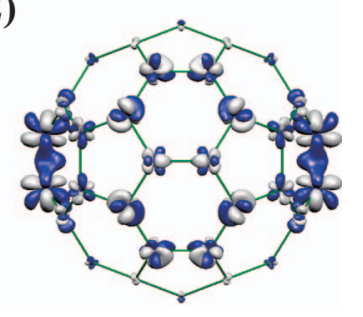

FIG. 4. Potential derivatives for the (a1) $h_{g}(2)$ and (b1) $h_{g}(8)$ modes and vibronic coupling densities for the (a2) $h_{g}(2)$ and (b2) $h_{g}(8)$ modes. The gray and blue indicate positive and negative, respectively. The isosurface value is 0.01 a.u. for the potential derivatives and $5 \times 10^{-6}$ a.u. for the VCDs. The orientation of $\mathrm{C}_{60}$ is the same as that of Fig. 1.

However, as we have discussed in Sec. IV B, the contributions to $\Delta \rho$ from the occupied orbitals are also important. The coefficients for the doubly occupied orbitals are negative because of the Coulomb repulsions between the electron in the LUMO and the other electrons in occupied orbitals. Since these orbitals have finite orbital coefficients of $s$ atomic orbitals and in-plane $p$ atomic orbitals, $\Delta \rho$ has negative polarized density. Among the orbitals other than the LUMO, $h_{g}(10)$ and $t_{1 g}(3)$ orbitals (Fig. 6 ) have the largest negative and positive coefficients, respectively. The densities of these orbitals can mix with each other because these orbitals have common representations in their direct products. Since the density of the $h_{g}(10)$ orbital overlaps the LUMO density, the Coulomb repulsion between the electrons occupied in the LUMO and the $h_{g}(10)$ orbitals is large. The Coulomb interactions give rise to an additional negative contribution in the $\Delta \rho$. Due to the decrease of the $h_{g}(10)$ density, the density around $\mathrm{C} 1, \mathrm{C} 2$, and $\mathrm{C} 8$ atoms where there is small singly occupied molecular orbital (SOMO) density also decreases. The negative density around $\mathrm{C} 1, \mathrm{C} 2$, and $\mathrm{C} 8$ is canceled by the $t_{1 g}(3)$ density. As a

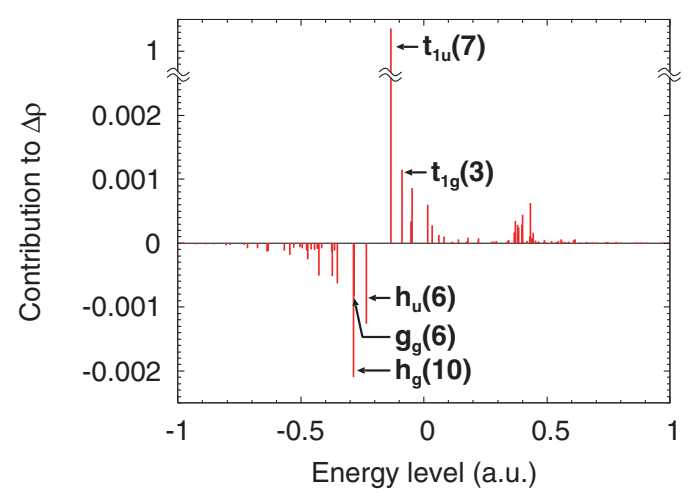

FIG. 5. Contribution of each Kohn-Sham orbital to $\Delta \rho . t_{1 u}(7)$ orbital is the LUMO.

(a)
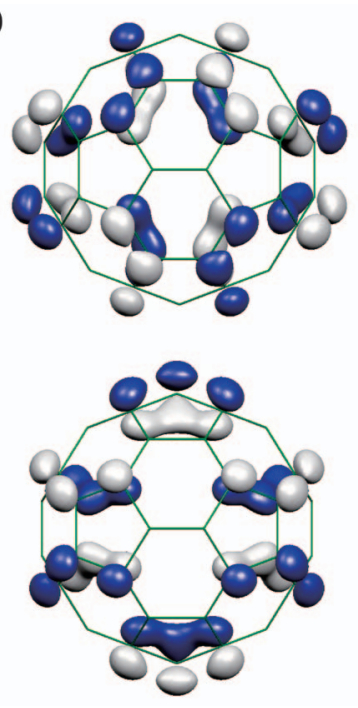

(b)
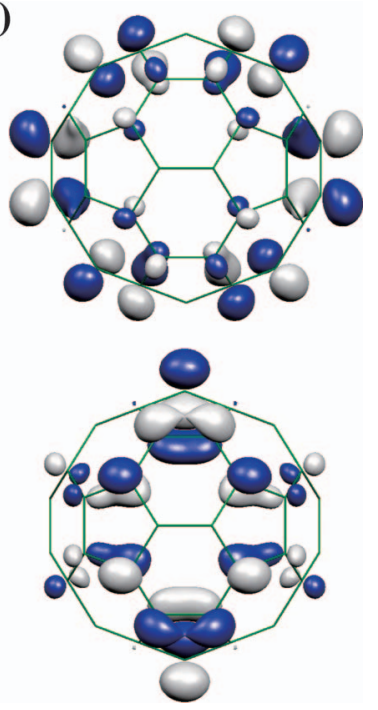

FIG. 6. (a) $h_{g}(10)$ orbitals and (b) $t_{1 g}(3)$ orbitals. The orientation of $\mathrm{C}_{60}$ is the same as that of Fig. 1.

result, $\Delta \rho$ is small around the $\mathrm{C} 1, \mathrm{C} 2$, and $\mathrm{C} 8$ atoms where the density of the $t_{1 u}(7)$ SOMO is small, whereas there are both positive and negative areas around the atoms where the SOMO density is large.

It is desired that the wavefunction of $\mathrm{C}_{60}^{-}$has the correct symmetry. Moreover, for the calculation of the VCCs, we must take into account the polarization of $\Delta \rho$. To fulfill both of them, a state-averaged post Hartree-Fock method could be one of the solutions. In the post Hartree-Fock calculation, we have to include not only frontier orbitals but also other orbitals which contribute to $\Delta \rho$.

\section{CONCLUSION}

In this work, we analyzed the order of the VCCs using the concept of the VCD. The coupling to the bending $h_{g}(2)$ mode is small because the displacement of the vibrational mode is perpendicular to the surface of the $\mathrm{C}_{60}$ cage and the VCD is localized on atom. On the other hand, the coupling to the stretching $h_{g}(8)$ mode is large due to the delocalization of the $\mathrm{VCD}$ on the $\mathrm{C} 8-\mathrm{C} 8^{\prime}$ bond. The polarization of the electron density difference is crucial for the couplings to the stretching modes. From the analysis of $\Delta \rho$, we found that the contribution from not only the frontier orbitals but also other orbitals are large.

\section{ACKNOWLEDGMENTS}

Theoretical calculations were partly performed using Research Center for Computational Science, Okazaki, Japan and the Supercomputer Laboratory of Kyoto University. This work was supported in part by the Global COE Program International Center for Integrated Research and Advanced Education in Materials Science (Grant No. B-09) of the Ministry of Education, Culture, Sports, Science and Technology (MEXT) of Japan, administered by the JSPS.

\footnotetext{
${ }^{1}$ I. B. Bersuker and V. Z. Polinger, Vibronic Interactions in Molecules and Crystals (Springer-Verlag, Berlin/Heidelberg, 1989).
} 
${ }^{2}$ I. B. Bersuker, The Jahn-Teller Effect (Cambridge University Press, Cambridge, 2005).

${ }^{3}$ C. M. Varma, J. Zaanen, and K. Raghavachari, Science 254, 989 (1991).

${ }^{4}$ M. Schluter, M. Lannoo, M. Needels, G. A. Baraff, and D. Tománek, Phys. Rev. Lett. 68, 526 (1992).

${ }^{5}$ O. Gunnarsson, H. Handschuh, P. S. Bechthold, B. Kessler, G. Ganteför, and W. Eberhardt, Phys. Rev. Lett. 74, 1875 (1995).

${ }^{6}$ N. Breda, R. A. Broglia, G. Colò, H. E. Roman, F. Alasia, G. Onida, V. Ponomarev, and E. Vigezzi, Chem. Phys. Lett. 286, 350 (1998).

${ }^{7}$ I. D. Hands, J. L. Dunn, and C. A. Bates, Phys. Rev. B 63, 245414 (2001).

${ }^{8}$ I. D. Hands, J. D. Dunn, and C. A. Bates, in Advances in Quantum Chemistry, edited by J. R. Sabin and E. Brändas (Elsevier, San Diego, 2003), Vol. 44, p. 335.

${ }^{9}$ I. D. Hands, J. L. Dunn, C. A. Bates, M. J. Hope, S. R. Meech, and D. L. Andrews, Phys. Rev. B 77, 115445 (2008).

${ }^{10}$ N. Manini, A. D. Carso, M. Fabrizio, and E. Tosatti, Philos. Mag. B 81, 793 (2001).

${ }^{11}$ M. Saito, Phys. Rev. B 65, 220508(R) (2002).

${ }^{12}$ T. Frederiksen, K. J. Franke, A. Arnau, G. Schulze, J. I. Pascual, and N. Lorente, Phys. Rev. B 78, 233401 (2008).

${ }^{13}$ J. Laflamme Janssen, M. Côté, S. G. Louie, and M. L. Cohen, Phys. Rev. B 81, 073106 (2010)

${ }^{14}$ C. Faber, J. Laflamme Janssen, M. Côté, E. Runge, and X. Blase, Phys. Rev. B 84, 155104 (2011).

${ }^{15}$ X. B. Wang, H. K. Woo, and L. S. Wang, J. Chem. Phys. 123, 051106 (2005).

${ }^{16}$ N. Iwahara, T. Sato, K. Tanaka, and L. F. Chibotaru, Phys. Rev. B 82, 245409 (2010)

${ }^{17}$ N. Iwahara, T. Sato, K. Tanaka, and L. F. Chibotaru, in Vibronic Interactions and the Jahn-Teller Effect: Theory and Applications, edited by M. Atanasov, C. Daul, and P. L.W. Tregenna-Piggott (Springer-Verlag, Berlin, 2012), p. 245.

${ }^{18}$ T. Sato, K. Tokunaga, and K. Tanaka, J. Chem. Phys. 124, 024314 (2006).
${ }^{19}$ K. Tokunaga, T. Sato, and K. Tanaka, J. Chem. Phys. 124, 154303 (2006).

${ }^{20}$ T. Sato, K. Tokunaga, and K. Tanaka, J. Phys. Chem. A 112, 758 (2008).

${ }^{21}$ T. Sato, K. Tokunaga, N. Iwahara, K. Shizu, and K. Tanaka, in The JahnTeller Effect: Fundamentals and Implications for Physics and Chemistry, edited by H. Köppel, D. R. Yarkony, and H. Barentzen (Springer-Verlag, Berlin, 2009), p. 99.

${ }^{22}$ K. Shizu, T. Sato, K. Tanaka, and H. Kaji, Appl. Phys. Lett. 97, 142111 (2010).

${ }^{23}$ K. Shizu, T. Sato, A. Ito, K. Tanaka, and H. Kaji, J. Mater. Chem. 21, 6375 (2011).

${ }^{24}$ T. Sato, K. Shizu, T. Kuga, K. Tanaka, and H. Kaji, Chem. Phys. Lett. 458, 152 (2008).

${ }^{25}$ T. Sato, K. Shizu, K. Uegaito, N. Iwahara, K. Tanaka, and H. Kaji, Chem. Phys. Lett. 507, 151 (2011).

${ }^{26}$ L. L. Boyle and Y. M. Parker, Mol. Phys. 39, 95 (1980).

${ }^{27}$ The VCCs for the $h_{g}$ modes $V_{h_{g}}(2)$ correspond to $-V_{E}$ in Ref. 34.

${ }^{28}$ Equation (4) is slightly different from the formula that we used in Ref. 16. The difference of VCCs between the two methods is less than $0.3 \%$ of the present VCCs.

${ }^{29}$ C. C. Chancey and M. C.M. O'Brien, The Jahn-Teller Effect in $C_{60}$ and Other Icosahedral Complexes (Princeton University Press, Princeton, 1997).

${ }^{30}$ A. D. Becke, J. Chem. Phys. 98, 5648 (1993).

${ }^{31}$ M. J. Frisch, G. W. Trucks, and H. B. Schlegel, et al., GAUSSIAN 09, Revision B.01, Gaussian, Inc., Wallingford, CT 2009.

${ }^{32}$ The vibronic couplings for the $a_{g}$ modes are taken from the data in Ref. 35 .

${ }^{33}$ See supplementary material at http://dx.doi.org/10.1063/1.4709611 for the VCDs for the $h_{g}(2)$ and $h_{g}(8)$ modes calculated with $\left|\Psi_{T_{1 u}}^{\mathrm{S}}\right\rangle$.

${ }^{34}$ M. C. M. O’Brien, Phys. Rev. 187, 407 (1969).

${ }^{35}$ V. P. Antropov, O. Gunnarsson, and A. I. Liechtenstein, Phys. Rev. B 48, 7651 (1993).

${ }^{36}$ D. S. Bethune, G. Meijer, W. C. Tang, H. J. Rosen, W. G. Golden, H. Seki, C. A. Brown, and M. S. de Vries, Chem. Phys. Lett. 179, 181 (1991). 